\title{
Skin Burning Sensation
}

National Cancer Institute

\section{Source}

National Cancer Institute. Skin Burning Sensation. NCI Thesaurus. Code C35297.

A sensation of dermal stinging or heat, not necessarily accompanied by redness or physical signs of skin irritation. 